\title{
Graphite from the viewpoint of Landau level spectroscopy: An effective graphene bilayer and monolayer
}

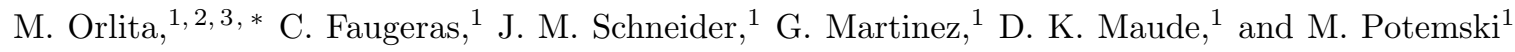 \\ ${ }^{1}$ Grenoble High Magnetic Field Laboratory, CNRS, BP 166, F-38042 Grenoble Cedex 09, France \\ ${ }^{2}$ Institute of Physics, Charles University, Ke Karlovu 5, CZ-121 16 Praha 2, Czech Republic \\ ${ }^{3}$ Institute of Physics, v.v.i., ASCR, Cukrovarnická 10, CZ-162 53 Praha 6, Czech Republic
}

(Dated: August 22, 2018)

\begin{abstract}
We describe an infrared transmission study of a thin layer of bulk graphite in magnetic fields up to $B=34 \mathrm{~T}$. Two series of absorption lines whose energy scales as $\sqrt{B}$ and $B$ are present in the spectra and identified as contributions of massless holes at the $H$ point and massive electrons in the vicinity of the $K$ point, respectively. We find that the optical response of the $K$ point electrons corresponds, over a wide range of energy and magnetic field, to a graphene bilayer with an effective inter-layer coupling $2 \gamma_{1}$, twice the value for a real graphene bilayer, which reflects the crystal ordering of bulk graphite along the $c$ axis. The $K$ point electrons thus behave as massive Dirac fermions with a mass enhanced twice in comparison to a true graphene bilayer.

PACS numbers: 71.70.Di, 76.40.+b, 78.30.-j, 81.05.Uw
\end{abstract}

Recent interest in graphene [1, 2], a truly twodimensional system with its simple, but nevertheless, for solids, unconventional electronic states, has focused attention on the properties of Dirac-like fermions in condensed matter physics in general. Whereas, twodimensional massless Dirac fermions [3, 4, 5], characteristic of graphene have been widely investigated, far fewer experiments have been devoted to massive Dirac fermions which are specific to a graphene bilayer [6, 7], which represents a further example of a two-dimensional system with a highly unusual band structure [8]. Perhaps surprisingly, Dirac dispersion relations can also be found in graphite, a three dimensional, bulk material which consists of Bernal-stacked weakly coupled graphene layers.

The standard Slonczewski-Weiss-McClure (SWM) model of electronic states in graphite [9, 10] predicts a complex form for the in-plane dispersion relation which changes considerably depending upon the value of the momentum $k_{z}$ in the direction perpendicular to the layers. Intriguingly, the SWM model predicts that in the vicinity of the $H$ point $\left(k_{z}=0.5\right)$ the in-plane dispersion is linear and thus resembles a Dirac cone. Such a dispersion has indeed been found in angle resolved photoemission spectroscopy [11, 12], tunneling spectroscopy [13, 14], as well as in Landau level (LL)spectroscopy [15]. The latter experiments, mainly focused on transitions between LLs whose energy scales as $\sqrt{B}$, are generally believed to exhibit far richer spectra in comparison to true graphene [16, 17, 18], reflecting the inherent complexity of the SWM model which includes no fewer than seven parameters [19, 20].

We show in this Letter that infrared magnetoabsorption spectra of graphite, measured over a wide range of the energy and magnetic field, can be interpreted in a very simple, transparent and elegant manner. Our results confirm, in agreement with theoretical considerations [21], that graphite can be viewed as an effective graphene monolayer and bilayer. This theoretical picture is derived using a drastically simplified SWM model, which includes only two parameters $\gamma_{0}$ and $\gamma_{1}$, describing the intra- and inter-layer tunneling respectively. In this simplified picture, the dominant contribution to the optical response is provided by the $H$ point, where electron states closely resemble graphene but with an additional double degeneracy, and by the $K$ point, where the energy spectrum resembles a graphene bilayer, but with an effective coupling of $2 \gamma_{1}$, twice enhanced compared to a real bilayer system.

Remarkably, using this simple graphene monolayer plus bilayer view of graphite, we are able to correctly reproduce the magnetic field evolution of all observed inter-LL transitions using only the SWM parameters $\gamma_{0}$ and $\gamma_{1}$, with values which perfectly match those derived from studies of real graphene monolayer and bilayer systems. Interestingly, the electronic states at $K$ point of graphite are found to mimic those of the graphene bilayer, but with a doubled value of the effective mass, so that they might be useful to further explore the interesting physics of massive Dirac fermions.

Thin samples for the transmission measurements were prepared by exfoliation of a natural graphite crystal as described in Ref. [15]. Some data is also presented for highly oriented pyrolytic graphite, which shows practically identical, although slightly less pronounced features, in the magneto-transmission spectra [22]. All experiments were carried out on macroscopic, roughly circular-shaped samples, of several millimeters in diameter. Measurements were performed in the Faraday configuration with the magnetic field applied along the $c$-axis of the graphite. All spectra were taken with non-polarized light. To measure the magneto-transmittance of the sample in the spectral range of $10-700 \mathrm{meV}$, the radiation of globar was delivered via light-pipe optics to the sample. The radiation, detected by a Si bolometer, placed directly 


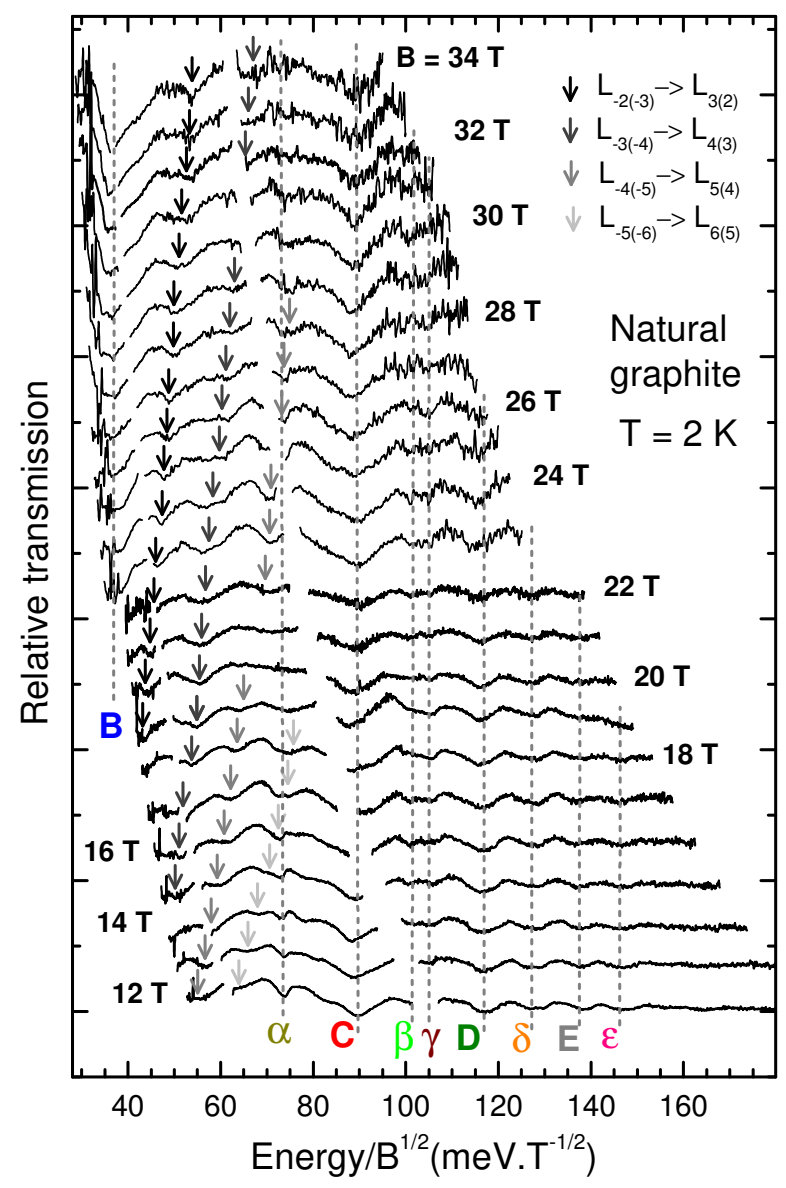

FIG. 1: (color online) Transmission spectra of a thin graphite layer as a function of the magnetic field in the interval $B=$ $12-34 \mathrm{~T}$. The plotted energy is scaled as $E / \sqrt{B}$ to emphasize the Dirac fermion-like features in spectra (indicated by dashed vertical lines). Arrows denote transitions arising at the $K$ point which evolve (nearly linearly) with $B$. Data above $B=$ $23 \mathrm{~T}$ were taken on second sample with a higher density of graphite flakes.

behind the sample and cooled down to a temperature of $2 \mathrm{~K}$, was analyzed by a Fourier transform spectrometer [15, 16]. The transmission spectra were normalized by the transmission of the tape and by the zero-field transmission, thus correcting for any magnetic field induced variations in the response of the bolometer. The missing parts of the transmission spectra, indicated by grey areas in Figs. 1 and 2, correspond to the spectral ranges where the tape is completely opaque.

Prior to presenting our experimental results, we outline a simple model of bulk graphite based on SWM model [9, 10]. Whereas the standard SWM model has seven tight-binding parameters $\gamma_{0}, \ldots, \gamma_{5}$, we limit ourselves here to only the most important hopping integrals $\gamma_{0}$ and $\gamma_{1}$. In other words, we consider only the parameters which are relevant for the nature of the band structure in a graphene monolayer and bilayer. In graphene, the intra-layer coupling parameter $\gamma_{0}$ is directly related to the Fermi velocity, $\tilde{c}=\sqrt{3} a_{0} \gamma_{0} /(2 \hbar)$, where the atomic distance is $a_{0}=0.246 \mathrm{~nm}$ [23], and in a graphene bilayer, the inter-layer coupling $\gamma_{1}$ gives an estimate for the mass of the charge carriers, $m=\gamma_{1} /\left(2 \tilde{c}^{2}\right)$.

Within our simplified approach, we calculate the band structure along the $H-K-H$ line of the Brillouin zone, i.e. for $-0.5<k_{z}<0.5$, which is essential for electrical and optical properties of bulk graphite. For the inplane dispersion of charge carries, we find [9, 10, 20, 24] that it has, for a given momentum $k_{z}$, the form of a graphene bilayer with an effective coupling $\lambda \gamma_{1}$, where $\lambda=2 \cos \left(\pi k_{z}\right)$ [20, 24].

In a magnetic field, we obtain the LL spectrum for each effective bilayer, i.e. for each momentum $k_{z}$ :

$$
\begin{aligned}
\varepsilon_{n, \mu}^{ \pm}= & \pm \frac{1}{\sqrt{2}}\left[\left(\lambda \gamma_{1}\right)^{2}+(2 n+1) E_{1}^{2}+\right. \\
& \left.\mu \sqrt{\left(\lambda \gamma_{1}\right)^{4}+2(2 n+1) E_{1}^{2}\left(\lambda \gamma_{1}\right)^{2}+E_{1}^{4}}\right]^{1 / 2}
\end{aligned}
$$

where \pm sign labels the electron( $(+)$ and hole(-) levels. LLs related to the touching electronic bands are obtained for $\mu=-1$ and those related to bands split-off in energy by an amount $\pm \lambda \gamma_{1}$ are represented by $\mu=1$. The touching bands can be in the parabolic approximation characterized by the mass $m=\lambda \gamma_{1} /\left(2 \tilde{c}^{2}\right)$. The inplane coupling $\gamma_{0}$ enters Eq. (1) via the Fermi velocity $\tilde{c}$ and directly influences the characteristic spacing of levels $E_{1}=\tilde{c} \sqrt{2 e \hbar B}$. Note, that our approach is a special case of the model used by Koshino and Ando 21], who in an analogous way calculated the spectrum of multilayer Bernal-stacked graphene with an arbitrary number of layers in an external magnetic field.

The joint density of states (initial and final states), essential in our magneto-optical experiments, has in the full as well as in our reduced SWM model singularities at two distinct points of the Brillouin zone, at the $K\left(k_{z}=0\right)$ and $H\left(k_{z}=0.5\right)$ points, where electrons and holes are located, respectively. Hence, the magneto-optical response of bulk graphite should be governed by transitions between LLs defined by Eq. (11) for $\lambda \rightarrow 2$ ( $K$ point) and $\lambda \rightarrow 0$ ( $H$ point). Notably, there is no singularity for $\lambda=1$, which corresponds to a real graphene bilayer. Consequently, bulk graphite should, in magneto-optical experiments, behave as a combination of a graphene bilayer with the effective coupling $2 \gamma_{1}$ and of a graphene monolayer, but with a twofold degeneracy $\varepsilon_{n,-1}^{ \pm}=\varepsilon_{n+1,1}^{ \pm}$, in addition to the usual twofold spin and valley degeneracies. The expected magneto-optical response of bulk graphite should therefore contain hole-related features whose energy evolves linearly with $\sqrt{B}$ originating in the vicinity of the $H$ point together with absorption lines whose energy evolves roughly linear with $B$ corresponding to electrons at the $K$ point.

The magneto-transmission spectra taken for magnetic fields $B=12-34 \mathrm{~T}$ on a thin layer of bulk graphite 


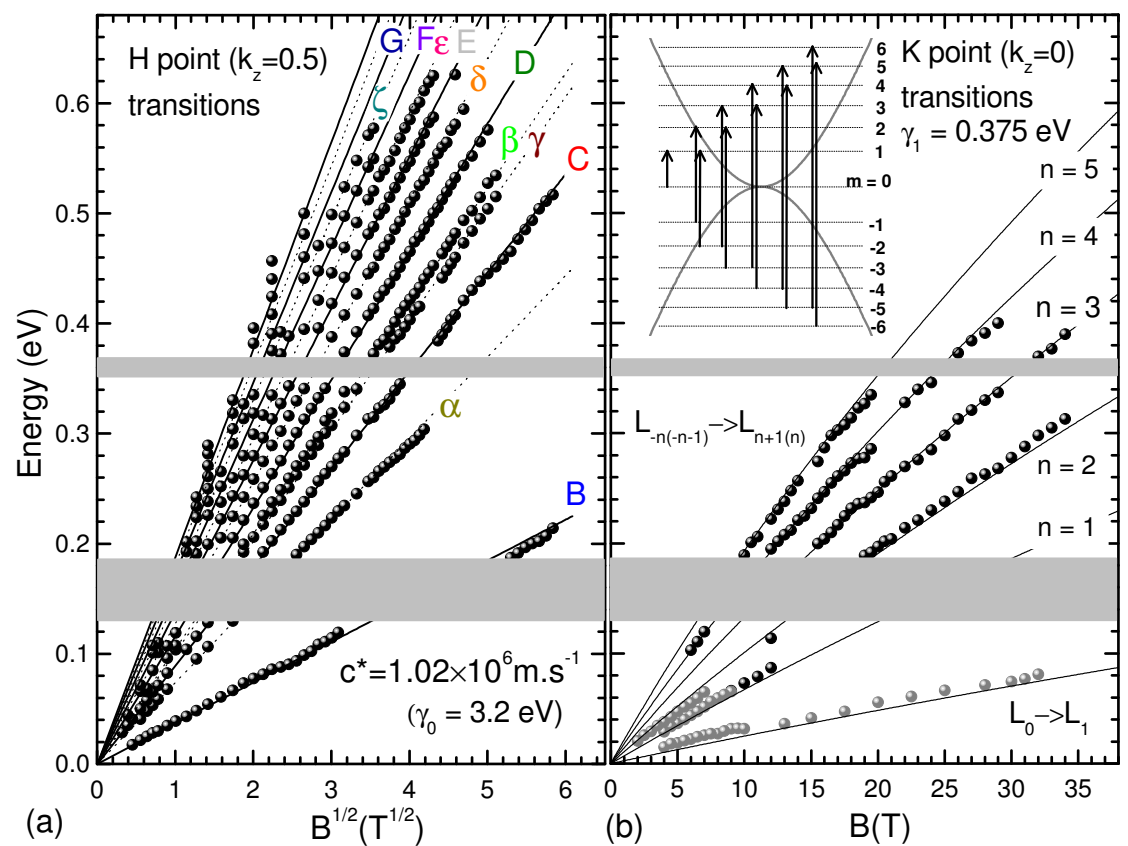

FIG. 2: (color online) (a): Positions of the absorption lines related to the $H$ point as a function of $\sqrt{B}$. The solid and dashed lines represent expected positions of absorption lines for $\tilde{c}=1.02 \times 10^{6} \mathrm{~m} / \mathrm{s}\left(\gamma_{0}=3.2 \mathrm{eV}\right)$. (b): $K$ point related absorption lines as a function of $B$. The solid lines show expected dipole allowed transitions in a graphene bilayer with an effective coupling $2 \gamma_{1}$ calculated using Eq. (1) for $\gamma_{0}=3.2 \mathrm{eV}$ and $\gamma_{1}=0.375 \mathrm{eV}$. Grey data points were taken on highly oriented pyrolytic graphite, which exhibit a behavior nearly identical to natural graphite [22]. The inset schematically shows the observed inter-band transitions in the effective bilayer. are presented in Fig. 1 The transmission is plotted as a function of energy divided by $\sqrt{B}$ to facilitate the identification of spectral features originating from around the $H$ and $K$ points. Plotted in this way, the transitions denoted by Roman and Greek letters, do not shift for spectra recorded at different magnetic fields. Thus they scale linearly with $\sqrt{B}$, see Fig. 2 a , and are related to the $H$ point. The second set of lines marked by vertical arrows shift with magnetic field and actually follow a nearly linear dependence with $B$, as can be seen in Fig. 2b.

The transitions following a $\sqrt{B}$ dependence, corresponding to massless holes around the $H$ point, have been thoroughly analyzed in our previous work [15, 22]. Whereas, the absorption lines denoted by Roman letters have their direct counterpart in spectra of true graphene [16, 17, 18, 25, 26, 27], the Greek lines are in principle dipole forbidden in a pure 2D system of Dirac fermions. Nevertheless, these transitions can be consistently explained with the same selection rule $\Delta n= \pm 1$, when the twofold degeneracy of LLs, $\varepsilon_{n,-1}^{ \pm}=\varepsilon_{n+1,1}^{ \pm}$, at the $H$ point of bulk graphite is properly considered. The Fermi velocity is extracted to be $\tilde{c}=(1.02 \pm 0.02) \times$ $10^{6} \mathrm{~m} / \mathrm{s}$, giving a rather precise measure of the in-plane hopping integral $\gamma_{0}=(3.20 \pm 0.06) \mathrm{eV}$ in bulk graphite, which is the only parameter needed to describe all absorption lines originating at the $H$ point.

Here we focus on transitions denoted by arrows in Fig. 1, whose energy evolves nearly linearly with $B$. Taking into account the selection rule $\Delta n= \pm 1$ and using the in-plane coupling estimated above to $\gamma_{0}=3.2 \mathrm{eV}$, we can interpret all the absorption lines in Fig. 2 $\mathrm{b}$ as dipoleallowed transitions in a graphene bilayer with an effective coupling $2 \gamma_{1}$ with $\gamma_{1}=(375 \pm 10) \mathrm{meV}$. The deduced value for $\gamma_{1}$ is in a very good agreement with results ob- tained for a real graphene bilayer [28, 29] as well as on bulk graphite [30, 31]. Our data follow well the theoretical predictions up to the highest energies, in contrast to the the deviation reported for a real graphene bilayer [7]. Hence, the electrons in the vicinity of the $K$ point can be described with a reasonable accuracy using the model of a graphene bilayer, but with coupling strength twice enhanced as compared to a true bilayer. The strength of the coupling $2 \gamma_{1}$ directly reflects the long-range Bernal stacking of graphite along the $c$ axis. The twice enhanced coupling in the effective bilayer can be understood using the example of semiconductor superlattices, which are three-dimensional but nevertheless strongly anisotropic systems resembling in some aspects the band structure of bulk graphite. The energy difference, $\Delta_{S A S}$, between the bonding and anti-bonding state in a symmetric double quantum well is simply half of the miniband width of the superlattice created from the same wells [32].

Our results also show that the parabolic approximation which is widely used for the touching electronic bands in a bilayer and which directly leads to LLs whose energy evolves linearly with magnetic field: $\varepsilon_{n,-}^{ \pm} \approx$ $\pm \hbar \omega_{c} \sqrt{n(n+1)}$ [8, 13] is a good approximation only in the vicinity of the charge neutrality point. As can be seen in Fig. 2(b), the small deviation from a linear dependence, predicted at higher energies by the simplified SWM model for an effective bilayer, is reproduced in our data. The bilayer character of $K$ point electrons also explains the non-linear evolution with $B$ of the magneto-reflection spectra published recently [19]. Nevertheless, within the parabolic approximation, we obtain an effective mass of $K$ point electrons in graphite of $m=\gamma_{1} / \tilde{c}^{2} \approx 0.063 m_{0}$ in good agreement with other cyclotron resonance experiments [33]. This mass is a factor 
of two higher when compared to a true bilayer [7].

While, only two tight-binding parameters $\gamma_{0}$ and $\gamma_{1}$ are required to obtain a reasonable description of the magneto-optical response of the $K$ point electrons over a wide range of energy and magnetic field, the influence of the remaining hopping integrals $\gamma_{2}, \ldots, \gamma_{5}$, merits some consideration. In general, additional coupling parameters should lift the electron-hole symmetry of the bilayer and the trigonal warping $\left(\gamma_{3}\right)$ should lead to a mixing of LLs, which in turn can give rise to additional dipole-allowed transitions.

The electron-hole asymmetry at the $K$ point should result in an energy difference of the transitions $\mathrm{L}_{-n} \rightarrow \mathrm{L}_{n+1}$ and $\mathrm{L}_{-n-1} \rightarrow \mathrm{L}_{n}$. Indeed, some evidence for this splitting is visible in the spectra, see e.g. the transition $n=3$ above $B \approx 20 \mathrm{~T}$ in Fig. 1] Nevertheless, this effect is relatively weak, comparable to the width of the absorption lines. The electron-hole asymmetry at the $K$ point of graphite seems to be somewhat weaker in comparison to the observed asymmetry in a true graphene bilayer [7, 29, 34].

The influence of the trigonal warping $\gamma_{3}$ on the magneto-optical response of the bilayer has been discussed by Abergel and Fal'ko [35]. They conclude that this parameter becomes important only in the limit of low magnetic fields giving rise to a completely new set of dipole-allowed transitions. Similar reasoning 36] explains the observation of numerous harmonics of the cyclotron resonance of electrons in bulk graphite at low magnetic fields [37, 38].

Our simplified model also neglects the hopping integral $\gamma_{2}$ which is directly responsible for the semi-metallic nature of bulk graphite. This parameter leads to a finite width of the doubly degenerate $E_{3}$ and consequently, the $k_{z}$-dependent LLs $n=0$ and $n=-1$ [24]. Assuming a finite and negative value of $\gamma_{2}$ [33], the $K$ point transition $\mathrm{L}_{-1} \rightarrow \mathrm{L}_{0}$ cannot be observed at any magnetic field at low temperatures, as the Fermi level remains close to the middle of $n=0$ and $n=-1$ Landau bands even in the quantum limit in graphite.

To conclude, the magneto-optical response of bulk graphite can, over a wide range of energy and magnetic field, be understood within a picture of an effective graphene monolayer and an effective bilayer with a coupling strength enhanced twice in comparison to a true graphene bilayer. This finding is in excellent agreement with predictions of a drastically reduced SWM model which retains only two tight-binding parameters, namely the in-plane and inter-plane coupling constants $\gamma_{0}$ and $\gamma_{1}$. It should be noted, that as the validity of the model is limited in the vicinity of the Fermi level, it is not useful, for example, for the interpretation of magnetotransport experiments. Nevertheless, bulk graphite remains a material of choice to study magneto-optical phenomena in systems with both massless as well as massive Dirac fermions.
Part of this work has been supported by EuroMagNET II under the EU contract, by the French-Czech Project Barrande No. 19535NF, by contract ANR-06-NANO-019 and by projects MSM0021620834 and KAN40010065.

* Electronic address: orlita@karlov.mff.cuni.cz

[1] A. K. Geim and K. S. Novoselov, Nature Materials 6, 183 (2007).

[2] A. H. Castro Neto et al., Reviews of Modern Physics p. to be published (2009).

[3] K. S. Novoselov et al., Nature 438, 197 (2005).

[4] Y. B. Zhang et al., Nature 438, 201 (2005).

[5] C. Berger et al., Science 312, 1191 (2006).

[6] K. S. Novoselov et al., Nature Phys. 2, 177 (2006).

[7] E. A. Henriksen et al., Phys. Rev. Lett. 100, 087403 (2008).

[8] E. McCann and V. I. Fal'ko, Phys. Rev. Lett. 96, 086805 (2006).

[9] J. C. Slonczewski and P. R. Weiss, Phys. Rev. 109, 272 (1958).

[10] J. W. McClure, Phys. Rev. 108, 606 (1957).

[11] S. Y. Zhou et al., Nature Phys. 2, 595 (2006).

[12] A. Grüneis et al., Phys. Rev. Lett. 100, 037601 (2008).

[13] G. Li and E. Andrei, Nature Phys. 3, 623 (2007).

[14] Yu. I. Latyshev et al., J. Phys.: Conf. Series 129, 012032 (2008).

[15] M. Orlita et al., Phys. Rev. Lett. 100, 136403 (2008).

[16] M. L. Sadowski et al., Phys. Rev. Lett. 97, 266405 (2006).

[17] Z. Jiang et al., Phys. Rev. Lett. 98, 197403 (2007).

[18] R. S. Deacon et al., Phys. Rev. B 76, 081406R (2007).

[19] Z. Q. Li et al., Phys. Rev. B 74, 195404 (2006).

[20] W. W. Toy, M. S. Dresselhaus, and G. Dresselhaus, Phys. Rev. B 15, 4077 (1977).

[21] M. Koshino and T. Ando, Phys. Rev. B 77, 115313 (2008).

[22] M. Orlita et al., J. Phys.: Condens. Matter 20, 454223 (2008).

[23] D. D. L. Chung, J. Mater. Sci. 37, 1475 (2002).

[24] K. Nakao, J. Phys. Soc. Jpn. 40, 761 (1976).

[25] M. L. Sadowski et al., Solid State Com. 143, 123 (2007).

[26] P. Plochocka et al., Phys. Rev. Lett. 100, 087401 (2008).

[27] M. Orlita et al., Phys. Rev. Lett. 101, 267601 (2008).

[28] L. M. Zhang et al., Phys. Rev. B 78, 235408 (2008).

[29] A. B. Kuzmenko et al., arxiv:0810.2400 (2008).

[30] A. Grüneis et al., Phys. Rev. B 78, 205425 (2008).

[31] A. B. Kuzmenko et al., Phys. Rev. Lett. 100, 117401 (2008).

[32] N. A. Goncharuk et al., Phys. Rev. B 71, 195318 (2005).

[33] N. B. Brandt, S. M. Chudinov, and Y. G. Ponomarev, Semimetals 1: Graphite and its Compounds, vol. 20.1 of Modern Problems in Condensed Matter Sciences (NorthHolland, Amsterdam, 1988).

[34] Z. Q. Li et al., Phys. Rev. Lett. 102, 037403 (2009).

[35] D. S. L. Abergel and V. I. Fal'ko, Phys. Rev. B 75, 155430 (2007).

[36] P. Nozières, Phys. Rev. 109, 1510 (1958).

[37] J. K. Galt, W. A. Yager, and H. W. Dail, Jr., Phys. Rev. 103, 1586 (1956).

[38] R. E. Doezema, W. R. Datars, H. Schaber, and A. Van Schyndel, Phys. Rev. B 19, 4224 (1979). 